\title{
A New Data Compression Technique for Event Based Program Traces
}

\author{
Andreas Knüpfer \\ Center for High Performance Computing \\ Dresden University of Technology, Germany \\ knuepfer@zhr.tu-dresden.de
}

\begin{abstract}
The paper presents an innovative solution to the problem of the very huge data sets that are regularly produced by performance tracing techniques - especially on HPC programs. It designs an adapted data compression scheme that takes advantage of regularities frequently found in program traces. Algorithms to reveal repetition patterns in a programs call structure and run time behavior are discussed in detail, solutions to some problems arising on practical application are addressed as well. Two examples demonstrate the capabilities of the approach and document its behavior. Finally, some thoughts are given regarding how the patterns revealed in the process of data compression may assist the automatic analysis of traces.
\end{abstract}

\section{Introduction}

While tracing is a well established method of program performance analysis, there is one critical part regularly encountered: the amounts of data generated. Of course, this problem is already addressed by instrumentation procedures. Furthermore, trace libraries do pay close attention on efficient encoding of trace data. In any case fact is that trace data sets grow bigger and bigger. On the one hand, this is caused by faster processors that produce more trace events per time. On the other hand, the amount of trace data is multiplied by the number of processes that work together to solve a problem. Especially in the High Performance Computing (HPC) field the trend to massive parallel computation causes immense trace data sizes.

At the same time the extractable information of a trace does not necessarily increase along with its size. So the question arises what the actual information is inside a trace. The answer to that question is tricky. But it is easy to see what non-information is included: ever repeating sequences produced by program loops over some essential parts of a program.

Such regularities are exploited to construct a data compression method specially suited for program traces. Of course, general data compression methods are being utilized for program traces, e.g. gzip and others, but compression ratios 1 for traces achieved by them grow rarely over a factor of five to ten.

\footnotetext{
${ }^{1}$ Compression ratio is defined as $\mathcal{K}:=$ original Size / compressed Size.
} 
The new approach was motivated by the work [234] about analysis and visualization of very large and highly parallel program traces of the local research group - especially in conjunction with the adaption of VAMPIR towards the ASCI project.

\section{Construction of Meta-events}

The given approach notices only events that denote function calls (enter events) or function returns (leave events) and ignores any other events like sending and receiving messages. Furthermore, the method takes only the current thread of a parallel program into account - thus for now it can be assumed that it has to deal with sequential programs only.

\section{$2.1 \quad$ Model}

By the restriction to enter and leave events - the most common classes of events in general - additional properties are assured: Following the procedural programming paradigm for every enter event $e$ there is exactly one associated leave event $l$. This relation is denoted as $e \sim l$. Given that the relation $<$ expresses the order of events in terms of time, for two pairs of associated events $e \sim l$ and $f \sim g$ the equivalence

$$
e<f<l \Leftrightarrow e<g<l
$$

is implied. This is called the stack property since it is caused by the LIFO scheme of the function calls. Now meta-events are defined as follows:

Definition 1. A collection of $n \in \mathbb{N}$ consecutive events $m:=\left\{e_{0}, e_{1}, \ldots e_{n-1}\right\}$ is called a meta-event of order $n$ if the implication $e_{i} \in m \Rightarrow \exists^{*} e_{j} \in m: e_{i} \sim e_{j}$ is satisfied for every $i, 0 \leq i<n$.

With this definition in mind it is easy to put up the three rules for constructing meta-events - see also Fig. 1.

1. The pair of an enter event $e$ and a leave event $l \sim e$ immediately following makes up a meta-event of order 2 .

2. A meta-event $m$ of order $n$, an enter event $e$ immediately before $m$ and a leave event $l \sim e$ immediately following $m$ make up a meta-event $m^{\prime}$ of order $n+2$.

3. Two meta-events $m$ and $m^{\prime}$ of order $n$ and $n^{\prime}$ make up a new meta-event of order $n+n^{\prime}$ if there are no events or meta-events between both. 


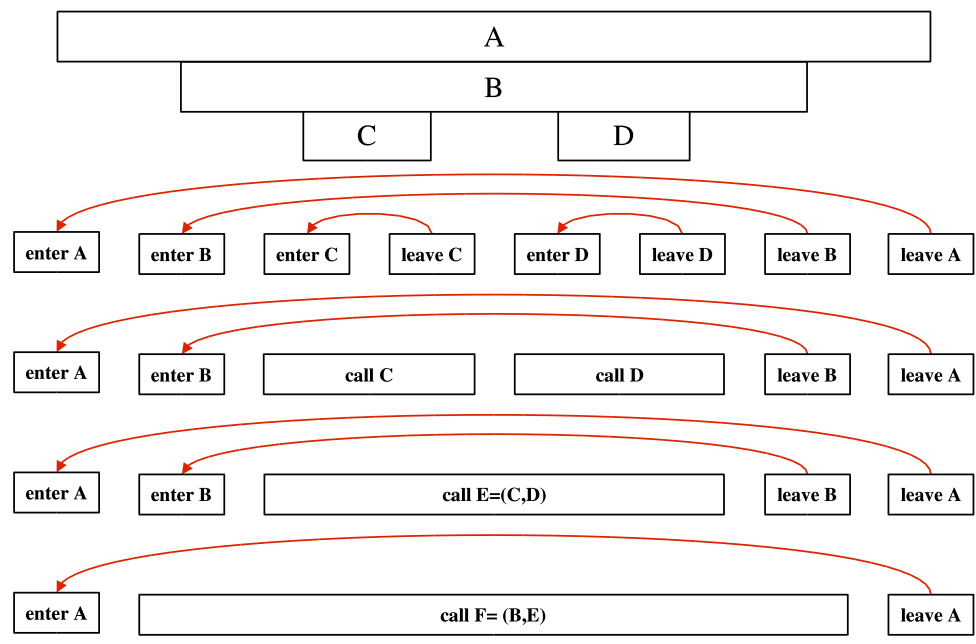

Fig. 1. Transformation from events to meta-events

\subsection{Implementation}

The first step for an application of the above scheme is to reveal the association $\sim$ between the single events. In fact, it is sufficient to be able to refer to the enter event $e \sim l$ that is associated with any given leave event $l$. This can be achieved very easily by extending the call stack representation of a trace library.

The second step is the application of the three construction rules for metaevents to the trace buffer - this works quite straight forward and it is done best by in-place substitution. The transformation algorithm has to traverse the event records in the order of creation and has to act only on leave records. For every possible situation exactly one of the three construction rules can be applied, compare Fig. 1.

1. If the current leave record references to the previous record as its associated one, then apply construction rule (1) - compare Fig. 1, (enter C, leave C).

2. If between the current leave record and its associated enter record there is exactly one meta-event record, apply construction rule (2) - compare Fig. [1 (enter B, call E, leave B). Note that by that time all leave records prior to the current one have already been processed.

3 . If there are $l \geq 2$ meta-events between associated events, apply construction rule (3) exactly $l-1$ times - compare Fig. 1 (enter B, call C, call D, leave B). After that branch 2) will be applicable.

Out of practical considerations it is suggested that the algorithm shall raise the order of the created meta-events only up to a certain order $N$ but not further. This implies that the sequence of events is transformed into a sequence of events and meta-events but in general not meta-events only! 


\subsection{Naming}

It is customary in tracing to refer to functions by tokens. A token is an integer number that can be stored with a fixed amount of bits. There is a bijective relation between the function set and the tokens set.

This naming scheme can be extended to meta-events as well, it might even share the same token set. The definition of a meta-event token must take the way of construction (rule 1,2,3) into account and the thereby referenced other events/meta-events resp. their tokens.

\section{Extending Meta-events to Event-Patterns}

Up to now the algorithm recognizes repetitions in a function call structure. In a second stage similarities in terms of execution times are to be detected: that is a meta-event that appears multiple times with "similar" vectors of timestamps.

\subsection{Comparison of Timestamp Vectors}

The timestamp vector of a meta-event of order $n$ is the vector

$$
\left(t_{0}, t_{1}, \ldots t_{n-1}\right) \in \mathbb{R}_{+}^{n}, t_{i} \leq t_{i+1} \forall i \in[0, n-2]
$$

of dimension $n$ that simply contains the timestamps of the atomic events. In order to reveal similarity 2 it is convenient to transform a timestamp vector into the equivalent representation of a time differences vector together with the very first time stamp value $t_{0}$ :

$$
\left(t_{0} ; d_{0}, d_{1}, \ldots d_{n-2}\right) \in \mathbb{R}_{+} \times \mathbb{R}_{+}^{n-1}, d_{i}:=t_{i+1}-t_{i} \geq 0 \forall i \in[0, n-2] .
$$

Now it is very simple to recognize two time differences vectors $d:=\left(d_{0}, \ldots d_{n-2}\right)$ and $e:=\left(e_{0}, \ldots e_{n-2}\right)$ as similar if all the corresponding vector components are similar. This can be tested by an absolute measure $a_{i}:=\left|d_{i}-e_{i}\right|$ or by a relative measure $r_{i}:=\left|1-\frac{e_{i}}{d_{i}}\right|$. The measure for the whole vectors is taken as maximum over all components $m_{\mathrm{abs}}:=\max _{0 \leq i<n-1}\left(a_{i}\right)$ and $m_{\mathrm{rel}}:=\max _{0 \leq i<n-1}\left(r_{i}\right)$. The suggestion is to combine the two ways of measuring: the two vectors are rated

$$
\text { similar }: \Leftrightarrow m_{\mathrm{abs}} \leq T_{\mathrm{abs}} \vee m_{\mathrm{rel}} \leq T_{\text {rel }}
$$

with two thresholds $T_{\text {abs }}$ and $T_{\text {rel }}$.

\subsection{Event-Patterns}

Where a meta-event represents several successive atomic events an event-pattern also respects the time behavior of the underlying part of a program.

\footnotetext{
${ }^{2}$ Testing for equality would be to strict.
} 
Definition 2. An event-pattern is defined as a 4-tuple of a meta-event of order $n$, a time differences vector (the representative vector) of dimension $n-1$ and the the absolute and relative thresholds $t_{a b s} \leq T_{a b s}$ and $t_{r e l} \leq T_{\text {rel }}$ for time differences vectors.

The transformation of meta-events into event-patterns is performed subsequent to the transformation of events into meta-events, i.e. it is only performed for the meta-events of maximal order. For each of the maximum-order meta-events the according time differences vector is compared with the representative vectors of all (so far defined) event-patterns based on the same meta-event. If similar eventpatterns are found choose the best fit, otherwise define a new event-pattern with the given time differences vector as the new representative vector.

\subsection{Over-All Data Compression Ratio}

In order to estimate the over-all data compression ratio trace data sizes are to be compared: First there is always a (relative small) number of general definition records that shall be ignored here: $l_{D}:=0$ bytes. For classic enter or leave events there is a fixed amount of $l_{E}:=18$ bytes storage space required 3 . So the total size for a trace file with $e$ events results in

$$
L_{0}:=l_{d}+l_{E} \cdot e \text { bytes. }
$$

An event-pattern is stored with $l_{P}:=30$ bytes regardless of its order $n$. For every event-pattern a definition record is required that needs $l_{E P}:=26+8 \cdot n$ bytes. That event-pattern references a tree of meta-event definitions. A single metaevent definition record requires $l_{M E}:=23$ bytes. The number of meta-event definitions $k$ depends on the order $n$ of the meta-event that forms the root of the tree and is

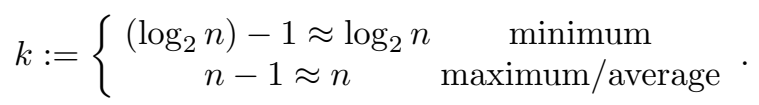

The total trace file size of a new-style trace file is then

$$
L_{1}:=l_{d}+l_{M E} \cdot k+l_{E P}+l_{P} \cdot \frac{e}{n} .
$$

With the sizes $L_{0}(e)$ and $L_{1}(e, n)$ the compression ratio achieved by the data compression approach can be predicted as

$$
\mathcal{K}:=\frac{L_{0}}{L_{1}}=\frac{l_{E} \cdot e}{l_{M E} \cdot k+l_{E P}+l_{P} \cdot \frac{e}{n}},
$$

see Fig. 2. For arbitrary high number of events and $k=$ const that results in

$$
\mathcal{K}:=\frac{L_{0}}{L_{1}} \longrightarrow \frac{l_{E}}{l_{P}} \cdot n=\frac{3}{5} \cdot n(e \rightarrow \infty, n=\text { const }),
$$

\footnotetext{
${ }^{3}$ All record sizes refer to the VTF3 binary trace file format [16].
} 

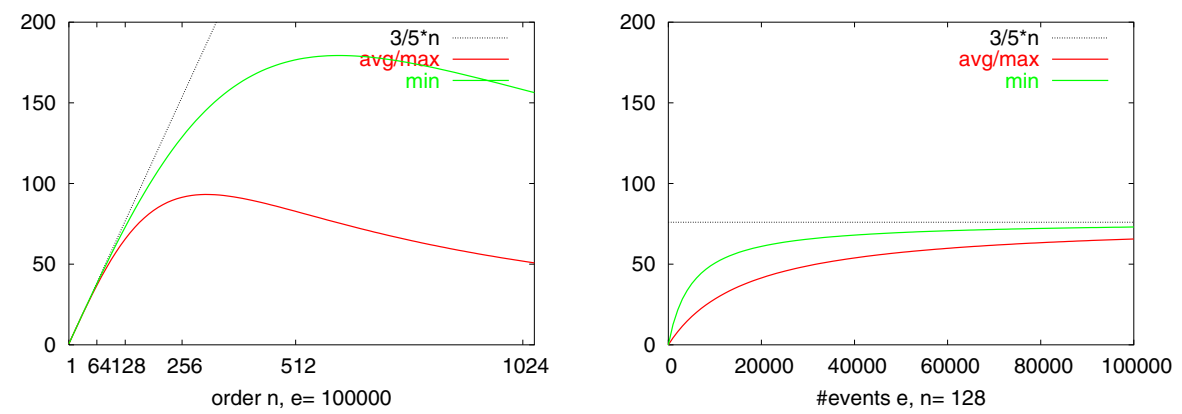

Fig. 2. Impact of the event count $e$ and the maximum order $n$ on the compression ratio $\mathcal{K}$. This shows idealistic behavior for a single repeated event-pattern

see Fig. 2b. The influence of $n$ (Fig. 2a) is more interesting: it occurs that $\mathcal{K}$ grows with $n$ up to a certain level. For higher values of $n$ and $e=$ const the compression ratio declines. The optimal $n$ grows with the number of events $e$. Thus $\mathcal{K}$ can be increased arbitrary by increasing $n$ as long as $e$ is big enough!

\section{Further Details}

There are two issues that are necessary to analyze at this stage. Both deal with problems that arise only in certain situations but which can destroy the usability of the whole approach, if not handled adequately.

\subsection{Correction Pairs}

The first problem is related to the limited regularity of execution times: Even if a program shows the most regular execution times there is always a source of irregularity present: the operating systems task scheduler. Without special precautions the algorithm would produce a set of event-patterns for an over and over repeated meta-event: one event-pattern shows the undisturbed execution of the meta-event, all the others have one or more components of the time differences vector influenced by the disturber. The data compression algorithm works nevertheless but suffers a higher definition overhead.

The solution to this problem is quite simple since a disturbed time differences vector does not differ much from the undisturbed version but in one (or few) components: the solution is to store only index and values of the disturbed components (called a correction pair) and refer to an existing event-pattern for all remaining ones.

\subsection{Name Spaces}

The second problem for general usability is connected with parallel programs. Unlike the classic approach tokens for meta-events and event-patterns must be 
supplied dynamically - it is impossible to provide a token for every possible meta-event. This means tokens have to be generated on demand. It is most undesirable to synchronize token generation among multiple processes so every process will create an own local set of tokens and in general these local sets will be incompatible with each other.

This can be corrected easily by a simple token translation: After all local tokens are known global tokens can be defined, then all occurrences of local tokens are replaced by their global counterparts in a post-processing step. This little extra effort prepares the compression algorithm to deal with non-sequential programs.

\section{$5 \quad$ Examples}

Now, two examples are presented in order to show the real world behavior of the algorithm. The examples shall address very regular and very irregular cases. For both the well known quick sort algorithm is utilized.

For the very regular case quick sort is called for sorting a rather small array as the body of a large loop, where the data to be sorted is always identical. This example can be understood as a general loop with anything in the body, where 'anything' is the same for all iterations. Figure 3 shows the compression ratios achieved for the regular example: $\mathcal{K}$ goes up to 45 where actually $4.5 \mathrm{MB}$ trace data is reduced to $100 \mathrm{~KB}$ ! The charts compare nicely to the theoretical result shown in Fig. 2. This plot is less smoothly because here the the given value for $N$ is an upper bound and not necessarily reached.

The second example uses quick sort to have a very large array of random data sorted once. This produces a huge and complex recursive call structure. Such a behavior might arise and is a rather bad case but after all there is still
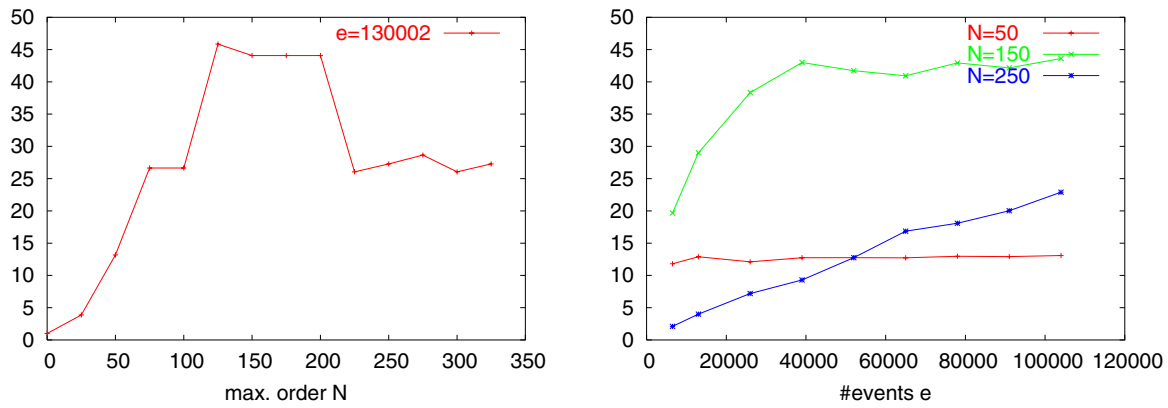

Fig. 3. Regular example: Influence of maximum order $N$ and event count $e$ on $\mathcal{K}$

$\overline{4}$ This can be combined with the merging of multiple process traces. 

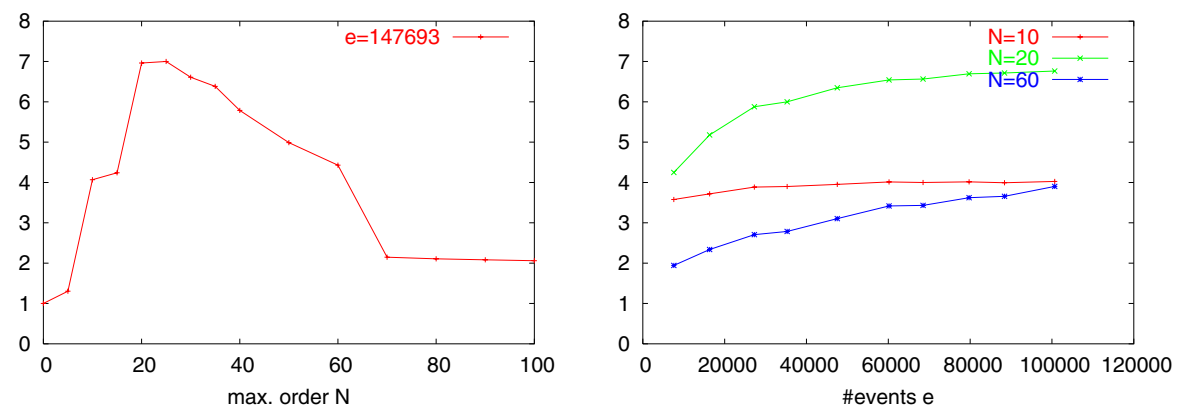

Fig. 4. Irregular example: Influence of maximum order $N$ and event count $e$ on $\mathcal{K}$
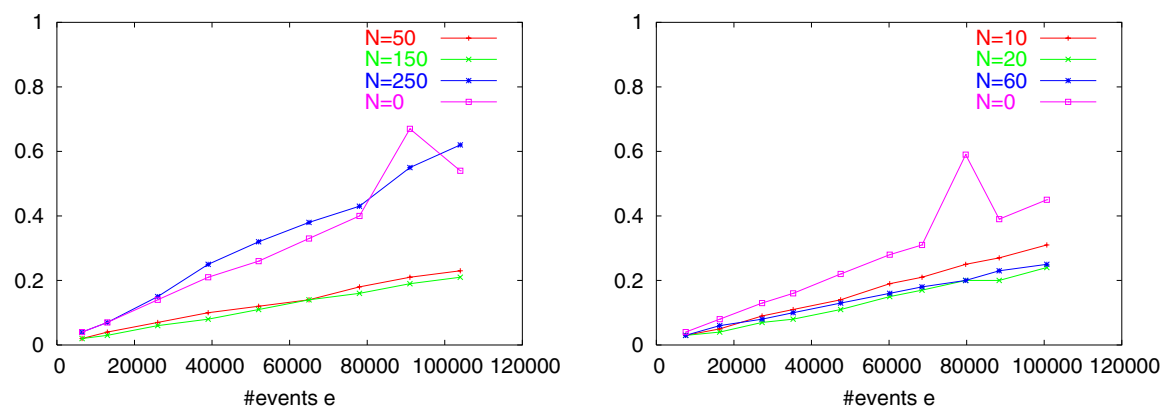

Fig. 5. Run times of regular and irregular experiments as measured on a PC AMD Athlon $1.4 \mathrm{GHz}$, disk writing performance $\approx 20 \mathrm{MB} / \mathrm{s}$

some kind of regularity left5. In Fig. 4 the charts of $\mathcal{K}$ depending on $N$ and $e$ are given for the irregular case. Of course, the result is not as good as in Fig. 3 but it reveals the ability of the algorithm to cope well with non-regular situations! And most important there are never values of $\mathcal{K}<1.0$ !

The run time consumed by the trace data compression algorithm might not be as important if it is performed post mortem. But the following figures shall proof that the algorithm can be used at trace time as well, i.e. trace data is compressed on-the-fly, no intermediate non-compressed output is generated at all. In Fig. 5a the run time durations are given for the experiments shown in Fig. 3 a, Fig. 5 b resp. for all experiments of Fig. 4 a. The case $N=0$ which means classical tracing without any compression is shown for comparison.

Obviously, the compression algorithm does not necessarily suffer a penalty for the increased computational effort. In fact, in the actual test cases it is faster than the classic tracing most of the time! This can be explained by the smaller amount of data to be written to disk after on-the-fly compression. Since this effect is heavily dependent on processor speed and disk I/O performance neither

\footnotetext{
${ }^{5}$ The worst case would be something like choosing the next function to call at random which is assumed to never ever happen in real life.
} 
classic tracing nor the version with on-the-fly compression can be said to be faster than the other in general.

\section{$6 \quad$ Future Plans}

Current work in connection with meta-events and event-patterns is focused on the analysis part of the performance investigation process. This is supposed to transfer the advantage of much smaller trace files to the tools that read and handle the data as well as to introduce the option to deal with meta-events and event-patterns directly.

So far there has only been discussion about compression but not about the decompression. Though, with a basic understanding of the compression scheme the according decompression is simple and works for every meta-event/eventpattern by itself. This is especially useful when accessing portions of a trace. A most elegant way to examine a compressed trace file would be to extract only the (few) event-patters that relate to the trace portion of interest! Thus the compression provides not only an advantage in terms of storage space but also in terms of access speed and response time during user interaction.

Another very interesting approach is using meta-events like more abstract functions, i.e. do not use functions as smallest entities but meta-events of certain (variable) sizes. This provides additional abstraction layers for trace analysis where so far there are merely two: statistics and fully detailed reproduction.

\section{Related Work}

Everything that is presented here is covered much more comprehensively in the German language diploma thesis [9]. There connections are drawn to string pattern matching [16 10 12, information encoding and general data compression algorithms [511,15] especially the Lempel-Ziv family.

Furthermore, for requirements and demands of trace analysis software tools [7/8 13 13 17,18 are referenced.

14 presents another very interesting data compression scheme for parallel program traces that is more or less orthogonal to the one presented here.

\section{References}

1. Jun-ichi Aoe. Computer Algorithms: String Pattern Matching Strategies. IEEE Computer Society Press, Los Alamitos, 1994.

2. H. Brunst, H.-Ch. Hoppe, and W.E. Nagel. Group Based Performance Analysis for Multithreaded SMP Cluster Applications. In Proceedings of Euro-Par2001, Manchester, UK, volume 2150 of LNCS, page 148ff. Springer-Verlag Berlin Heidelberg New York, August 2001.

3. H. Brunst, H.-Ch. Hoppe, W.E. Nagel, and M. Winkler. Performance Otimization for Large Scale Computing: The Scalable VAMPIR Approach. In Proceedings of ICCS2001, San Francisco, USA, volume 2074 of LNCS, page 751ff. Springer-Verlag Berlin Heidelberg New York, May 2001. 
4. H. Brunst, W.E. Nagel, and S. Seidl. Performance Tuning on Parallel Systems: All Problems Solved? In Proceedings of PARA2000 - Workshop on Applied Parallel Computing, volume 1947 of LNCS, pages 279-287. Springer-Verlag Berlin Heidelberg New York, June 2000.

5. T.H. Cormen, C.E. Leiserson, and R. L. Rivest. Introduction to Algorithms. MIT Press, 1990.

6. G. Davies and S. Bowsher. Algorithms for Pattern Matching. Software - Practice and Experience, June 1986.

7. R. Jain. The Art of Computer Systems Performance Analysis. John Wiley \& Sons, Inc., 1991.

8. R. Klar et al. Messung und Modellierung paralleler und verteilter Rechnersysteme. B.G.Teubner, Stuttgart, 1995.

9. A. Knuepfer. Analyse von Programmspuren: Entwurf und Implementierung eines effizienten Algorithmus zur Datenreduktion. Technical Report ZHR-R-0202, Zentrum für Hochleistungsrechnen, TU-Dresden, June 2002.

10. D.E. Knuth. The Art of Computer Programming: Fundamendal Algorithms, volume 1. Addison Wesley Longman, zweite edition, 1997.

11. D.E. Knuth. The Art of Computer Programming: Sorting and Searching, volume 3. Addison Wesley Longman, zweite edition, 1998.

12. D.E. Knuth, J.H. Morris, and V.R. Pratt. Fast Pattern Matching in Strings. SIAM Journal of Computing, 061977.

13. B. Mohr. Ereignisbasierte Rechneranalysesysteme zur Bewertung paralleler und verteilter Systeme. VDI Verlag, 1992.

14. O.Y. Nickolayev, P.C. Roth, and D.A. Reed. Real-time statistical clustering for event trace reduction. The International Journal of Supercomputer Applications and High Performance Computing, 11(2):144-159, Summer 1997.

15. R. Sedgewick. Algorithmen. Addison Wesley, 1991.

16. S. Seidl. VTF3 - A Fast Vampir Trace File Low-Level Library. personal communications, May 2002.

17. F. Wolf. EARL - Eine programmierbare Umgebung zur Bewertung paralleler Prozesse auf Message-Passing-Systemen. Technical report, Forschungszentrum Jülich GmbH, June 1998. JÜL-3551.

18. F. Wolf and B. Mohr. Automatic Performance Analysis of SMP Cluster Applications. Technical report, Forschungszentrum Jülich GmbH, August 2001. FZJZAM-IB-2001-05. 